Original paper

\title{
Simulated four-dimensional CT for markerless tumor tracking using a deep learning network with multi-task learning
}

\author{
Shinichiro Mori ${ }^{\text {a, }}$, Ryusuke Hirai ${ }^{\mathrm{b}}$, Yukinobu Sakata ${ }^{\mathrm{b}}$ \\ ${ }^{a}$ Research Center for Charged Particle Therapy, National Institute of Radiological Sciences, Inage-ku, Chiba 263-8555, Japan \\ ${ }^{\mathrm{b}}$ Corporate Research and Development Center, Toshiba Corporation, Kanagawa 212-8582, Japan
}

\section{A R T I C L E I N F O}

\section{Keywords:}

Targeted radiation therapy

Three-dimensional imaging

Computer generated

Machine learning

\begin{abstract}
A B S T R A C T
Introduction: Our markerless tumor tracking algorithm requires 4DCT data to train models. 4DCT cannot be used for markerless tracking for respiratory-gated treatment due to inaccuracies and a high radiation dose. We developed a deep neural network (DNN) to generate 4DCT from 3DCT data.

Methods: We used 2420 thoracic 4DCT datasets from 436 patients to train a DNN, designed to export 9 deformation vector fields (each field representing one-ninth of the respiratory cycle) from each CT dataset based on a 3D convolutional autoencoder with shortcut connections using deformable image registration. Then 3DCT data at exhale were transformed using the predicted deformation vector fields to obtain simulated 4DCT data. We compared markerless tracking accuracy between original and simulated 4DCT datasets for 20 patients. Our tracking algorithm used a machine learning approach with patient-specific model parameters. For the training stage, a pair of digitally reconstructed radiography images was generated using 4DCT for each patient. For the prediction stage, the tracking algorithm calculated tumor position using incoming fluoroscopic image data. Results: Diaphragmatic displacement averaged over 40 cases for the original 4DCT were slightly higher $(<1.3$ $\mathrm{mm}$ ) than those for the simulated 4DCT. Tracking positional errors (95th percentile of the absolute value of displacement, "simulated 4DCT" minus "original 4DCT") averaged over the 20 cases were $0.56 \mathrm{~mm}, 0.65 \mathrm{~mm}$, and $0.96 \mathrm{~mm}$ in the $\mathrm{X}, \mathrm{Y}$ and $\mathrm{Z}$ directions, respectively.

Conclusions: We developed a DNN to generate simulated 4DCT data that are useful for markerless tumor tracking when original 4DCT is not available. Using this DNN would accelerate markerless tumor tracking and increase treatment accuracy in thoracoabdominal treatment.
\end{abstract}

\section{Introduction}

Currently, radiotherapy targeting the thoracoabdominal region incorporates intrafractional motion by using 4D imaging [1]. The intrafractional position changes caused by respiration are not always the same in the same respiratory time point from cycle to cycle, making it difficult to predict exact target position even when four-dimensional computed tomography (4DCT) data are acquired before treatment. To solve this problem, image guidance techniques (image-guided radiotherapy: IGRT) are routinely used to monitor tumor position in realtime, including using fluoroscopic imaging [2] or magnetic resonance imaging [3] during irradiation to improve treatment accuracy $[1,4,5]$.

In 2015, our center began using gated carbon-ion pencil beam scanning (C-PBS) treatment using fluoroscopy-based markerless tracking for the thoracoabdominal area, which is subject to respiratory motion [6]. At that time, this markerless tracking technique used fluoroscopic image registration and machine learning approaches; however, it required preparation of reference data before irradiation. To solve this problem, we used a machine learning approach with treatment planning 4DCT for each patient and used it on the fluoroscopic image data acquired during irradiation to track the tumor position [7-9]. As we know, there is no markerless tracking algorithm with a single machine learning model file that can be applied to every patient; therefore, treatment planning 4DCT for each patient was required.

Other researchers introduced similar methodology [10,11]. A 4DCT dataset is mandatory for these techniques, and the tracking accuracy strongly depends on the quality of the data. However, not all treatment centers still perform planning 4DCT due to the higher radiation dose and longer examination time involved. Moreover, when there are inconsistencies between a point in the respiratory cycle and tumor

\footnotetext{
* Corresponding author.

E-mail address: mori.shinichiro@qst.go.jp (S. Mori).
} 
position, geometrical error may be generated in the 4DCT image. One study reported approximately $90 \%$ of all patient studies showing some degree of artifact, including blurring, duplication, overlapping, and incomplete imaging when using a conventional multidetector CT scanner [12].

Other approaches to this problem are to generate 4DCT image datasets from 3DCT inhalation and exhalation images [11], where a deformation vector field is generated from cine MRI or fluoroscopic images and 3DCT data are warped using the deformation vector field as a function of the respiratory phase $[13,14]$.

We have developed a new deep neural network (DNN) for generating 4DCT data (simulated 4DCT) from 3DCT data and have evaluated its accuracy in markerless lung tumor tracking.

\section{Materials and methods}

\subsection{Image acquisition}

A total of 436 patients with tumors of the lung receiving C-PBS treatment participated at our center. The treatment planning CT was performed under free-breathing conditions using a 320-detector CT (Aquilion One Vision ${ }^{\circledR}$, Canon Medical Systems, Otawara, Japan). Since our treatment facility used orthogonal beam ports $\left(0^{\circ}\right.$ and $\left.90^{\circ}\right)$, the treatment couch requires to be rotated around its long axis to extend the range of angles and/or changes patient position to prone/supine. Therefore, a single 4DCT data set was acquired for 388 patients and two 4DCT data sets (different couch angle and/or patient pose) were acquired for 48 patients. CT imaging conditions were a tube voltage of 120 $\mathrm{kV}$, slice collimation of $270 \times 0.5 \mathrm{~mm}$ or $320 \times 0.5 \mathrm{~mm}$ in volumetric cine mode (4DCT mode). To cover the entire lung region, we repeated 4DCT imaging contiguously. Tube current was automatically adjusted to obtain clinically acceptable image quality. Reconstruction parameters were field of view (FOV) of $500 \mathrm{~mm}^{2}$ and slice thickness of $2.0 \mathrm{~mm}$. The 4DCT dataset was subdivided into 10 sections (T00 = peak inhalation, T50 = peak exhalation).

Since our treatment center uses orthogonal beam ports $\left(0^{\circ}\right.$ and $\left.90^{\circ}\right)$, the treatment couch needed to be rotated around its long axis ( $\phi$ : standard International Electrotechnical Commission [IEC] tabletop rolling angle) to extend the range of angles $\left(< \pm 20^{\circ}\right)$. Patients requiring irradiation in the posteroanterior direction were positioned prone.

\subsection{Fluoroscopic image data}

Two oblique X-ray fluoroscopic units consisting of a pair of dynamic flat panel detectors (DFPDs) and X-ray tubes are in the treatment room. DFPD1 and DFPD2 are on the right and left sides of the vertical irradiation port at $35^{\circ}$ and $325^{\circ}$, respectively, and the X-ray tubes are under the floor. The DFPD is an indirect type (PaxScan 3030+, Varian Medical Systems, Palo Alto, CA) with an imaging area size of $296 \times 296 \mathrm{~mm}$, pixel size of $388 \mu \mathrm{m}(2 \times 2$ binning mode using the original pixel size of $194 \mu \mathrm{m}$ for fluoroscopic mode). Distance from the room isocenter and source-image receptor distance are $1690 \mathrm{~mm}$ and $2390 \mathrm{~mm}$, respectively, allowing acquisition of an image size of approximately $210 \times$ $210 \mathrm{~mm}$ at the room isocenter. Images are acquired at $15 \mathrm{fps}$. Fluoroscopic imaging is acquired during treatment beam irradiation. The total imaging time depends on the treatment volume, respiratory pattern, and other patient factors. The quality of fluoroscopic images was improved by applying image processing [15].

\subsection{Network architecture}

Our DNN for 4DCT simulation generation was designed to export nine deformation vector fields from a single CT dataset based on a 3D convolutional autoencoder (AE) with shortcut connections [16] (Fig. 2a). The AE has encoder (to generate the representation with reduced spatial dimensions) and decoder (to restore the original spatial dimensions of the representation) functions. It is often used to restore image quality and segmentation [17-20] and to obtain performance better than that provided by a conventional neural network. To avoid a vanishing gradient in the deep architecture, one solution is to use shortcut connections [21-23]. As an example, U-net uses a shortcut connection between the encoder and decoder blocks [22]. However, since our DNN takes input of a single dataset (CT image) and output nine different datasets (deformation vector fields), shortcut connections are applied to every two convolutional layers (residual block), which involves two sets of batch normalization and Leaky Rectified Linear Units (ReLU) and 11 convolutional layers (kernel size of $3 \times 3 \times 3$ ), plus one convolutional layer (kernel size of $1 \times 1 \times 1$ ) to adjust the number of channels, and then the tensors in respective channels from both outputs are added by applying element-wise addition [11] (Fig. 2b).

For the encoding process, we added a convolutional layer (kernel and stride size were $3 \times 3 \times 3$ pixels and $1 \times 1 \times 1$ pixels, respectively, the number of output channels $=64$ ) in the first layer (blue box in Encoder block in Fig. 1a). Four sets of the residual block were added, and max pooling layer was applied to reduce to the data to half their original size (both kernel and stride size were $2 \times 2 \times 2$ pixels) while increasing the number of convolutional output channels $(64,128,256$, and 512). A fifth residual block with 1024 convolutional output channels was added. This network structure is referred to as the "encoder block."

For the decoding process, three deconvolutional layers (kernel and stride size were $3 \times 3 \times 3$ pixels and $2 \times 2 \times 2$ pixels, respectively) were resized to double the width/height and half the number of channels in the tensors, and a residual block was added, decreasing the number of convolutional/deconvolutional output channels to 512, 256, and 128. Generally, this decoding process restores the same dimensions as those of the 3DCT; however, our network exported deformation vector field data (data dimension was $64 \times 64 \times 40$ pixels, the number of channels was 3), which do not require the same dimensions as those of the 3DCT; therefore, a deconvolutional layer was not added after the third residual layer. A residual block with a convolutional output channel of 64 and a convolutional layer (kernel size $=1 \times 1 \times 1$, with three output channels) were added to the end of the network. We defined this network structure as the "decoder block." Nine decoder blocks were connected in parallel after the encoder block to export nine deformation vector fields (T00, ... $\mathrm{T} 40, \mathrm{~T} 60, \ldots \mathrm{T} 90)$.

\subsection{Network training}

\subsubsection{Training data}

Since the number of CT slices differs among patients, the number of slices was limited to 160 to input the same dimensional data. The original 4DCT dimension of $512 \times 512 \times 160$ pixels was resized to 128 $\times 128 \times 80$ pixels. If the treatment couch was rotated, CT images were rotated to an angle of $0^{\circ} .4 \mathrm{DCT}$ data were shifted $\pm 10 \mathrm{~mm}$ randomly in 1-mm steps along the anterior-posterior and/or lateral directions (data augmentation), This was repeated five times per patient to increase the amount of data. The input data into the DNN were 3DCT simulated from 4DCT at T50.

For output data (ground-truth), the deformation vector field from CT at T50 to CT at T $n$ was calculated by applying B-spline-based deformable image registration (DIR) [24]. Then the nine deformation vector field files were defined as the ground-truth data (Fig. 1a). The deformation vector field data dimension of $128 \times 128 \times 80$ pixels with 3 channels was resized to the same size of the network output data size $(=64 \times 64$ $\times 40$ pixels with 3 channels).

A total of 2420 datasets from 436 patients $(=388$ patients $\times 5$ augmentation $\times 1$ pose +48 patients $\times 5$ augmentation $\times 2$ poses) were prepared for training. All image preprocessing was performed using a commercial programming environment (MATLAB R2019a ${ }^{\circledR}$, Mathworks, Natick MA, USA). 


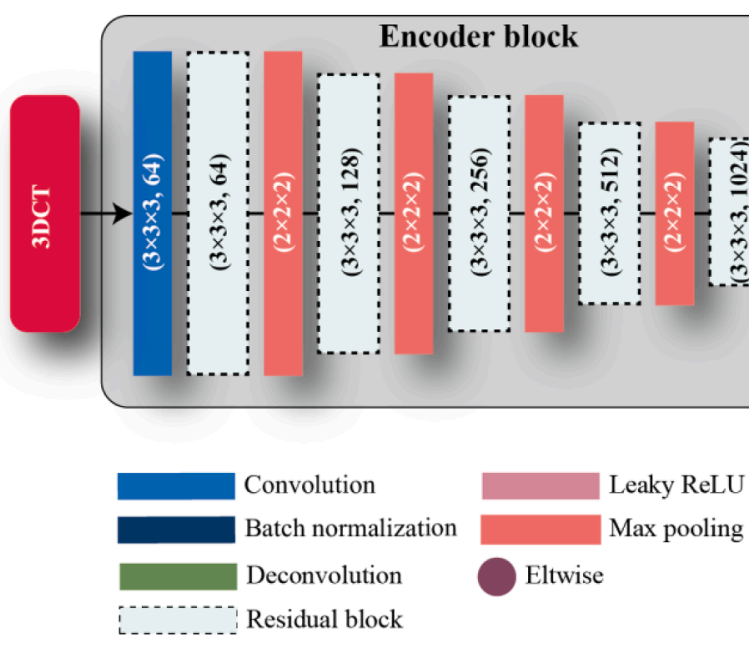

(a)

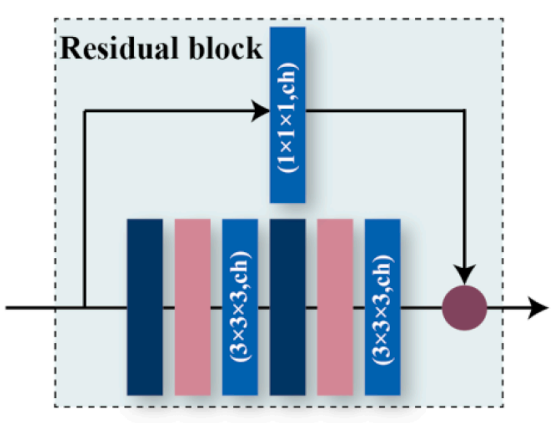

(b)
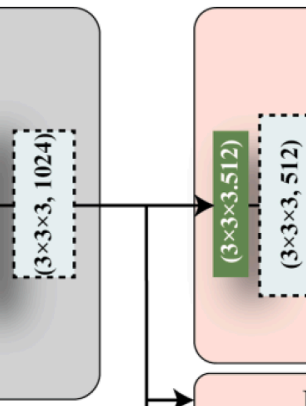

Decoder block (T00)
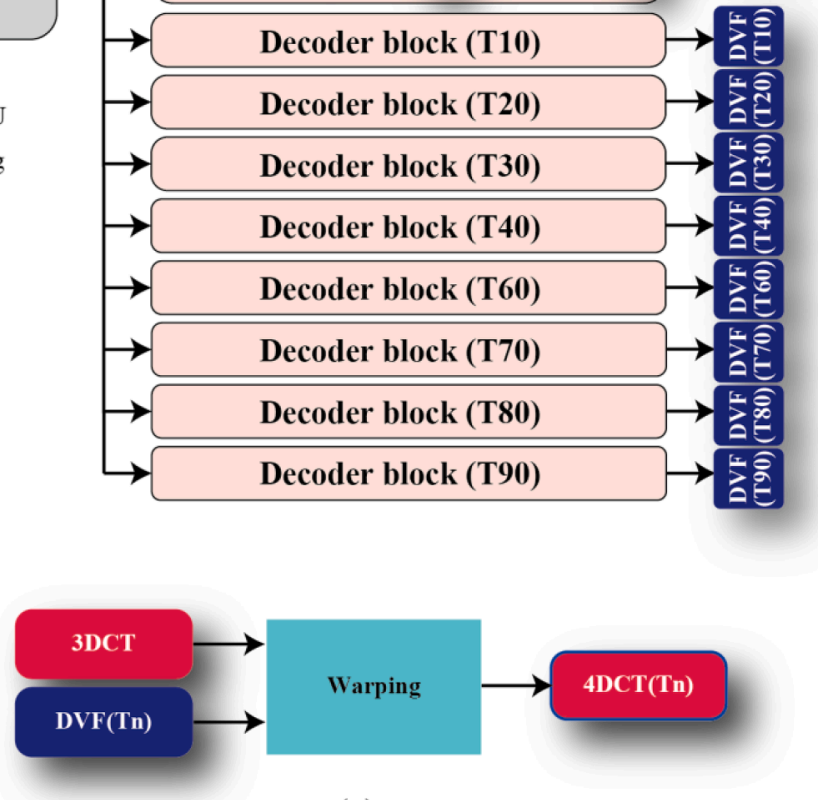

(c)

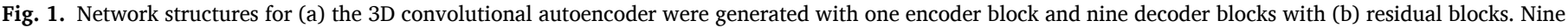

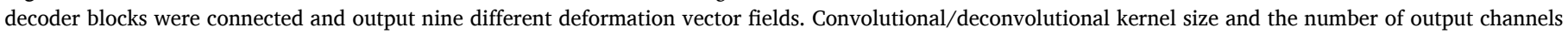

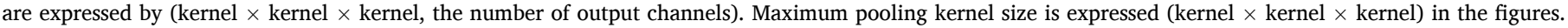

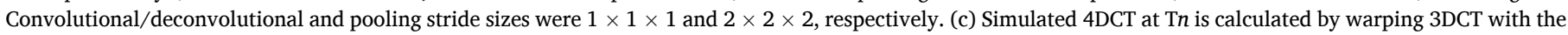
deformation vector field at Tn. Abbreviations: Eltwise = element-wise addition; ReLU = rectified linear units;

\subsubsection{Parameter optimization}

Since the DNN was trained to export nine deformation vector field files close to those of the ground-truth deformation vector fields, we used a multitask learning technique [25]. An optimization procedure was performed for 20,000 iterations with a batch size of 1 using stochastic gradient descent (SGD) to minimize the respective nine mean square errors (MSE) of differences between the output deformation vector field at $\mathrm{T} n$ through the DNN and the ground-truth deformation vector field at $\mathrm{T} n$. The learning rate, clip norm, weight decay, and momentum were $0.001,200,10^{-6}$, and 0.9 , respectively. The deep learning framework "TensorFlow" was used in a 64-bit environment (Ubuntu 18, Canonical UK Ltd.) and a single GPU on a board (NVIDIA Quadro P5000 $尺$, NVIDIA Corporation, Santa Clara CA, USA), which is equipped with 2560 compute unified device architecture (CUDA) cores and has 16 GB of memory.

Since we sent the input 3DCT data and the nine output deformation vector field datasets into the GPU, the training process with the GPU did not work due to the limitation of GPU memory. Therefore, unified virtual memory (UVM) implemented on CUDA was applied. The UVM function managed swapping of GPU memory with the host (CPU) and fetched data from the host to the GPU to allocate more memory than usually available on a GPU.

\subsection{Generating $4 D C T$}

3DCT data at full exhale were imported into the DNN, and nine deformation vector fields were predicted. Then, to obtain images with better spatial resolution, the dimensions of the 3DCT and deformation vector fields were generated by up-sampling to $512 \times 512 \times 160$ pixels. Input 3DCT data were transformed using the predicted deformation vector fields for the respective sections of the respiratory cycle to obtain simulated 4DCT datasets (Fig. 1c).

\subsection{Evaluation}

\subsubsection{Diaphragmatic displacement}

Diaphragmatic displacements in both the original 4DCT and the simulated 4DCT were compared in their respective phases for 40 randomly selected patients; these data were not used for the training. A measurement point was set on the dome of the diaphragm.

\subsubsection{Markerless tracking accuracy}

We compared the markerless tumor tracking positional accuracy by using original and simulated 4DCT data. The markerless tracking algorithm uses a machine learning approach. The model parameters is required for every patient using 4DCT for respective patients $[7,8]$.

For the training stage, the gross tumor volume (GTV) contour was input to 4DCT at T50 on the treatment planning system by an oncologist, and propagated to other sections of the respiratory cycle by DIR. A pair of digitally reconstructed radiography (DRR) images as a function of respiratory phase section (4D-DRR) was generated by projecting voxels along the fluoroscopy beam path. Tumor positions on 4DCT were 


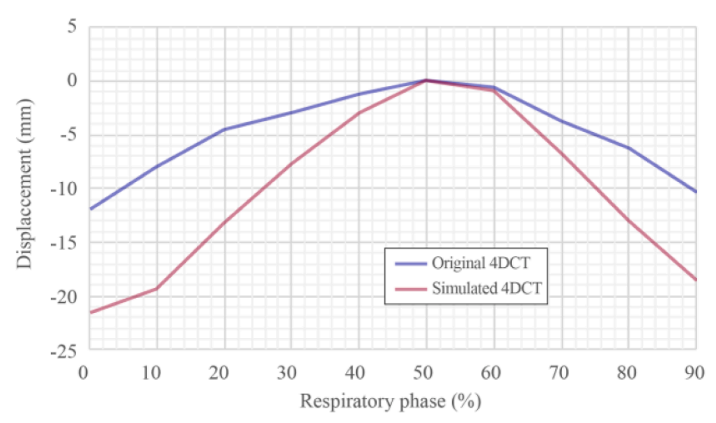

(a)

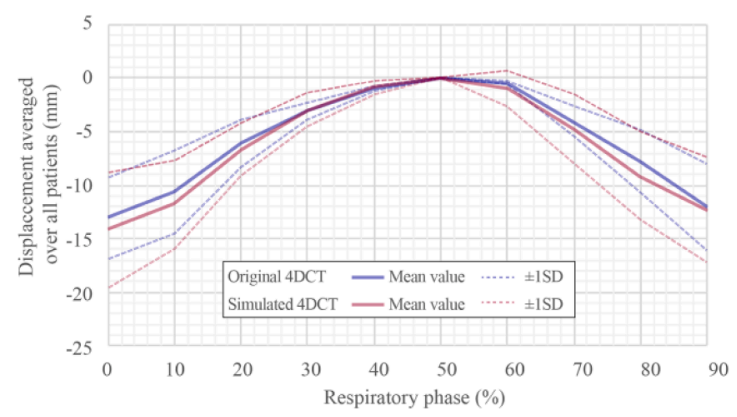

(c)

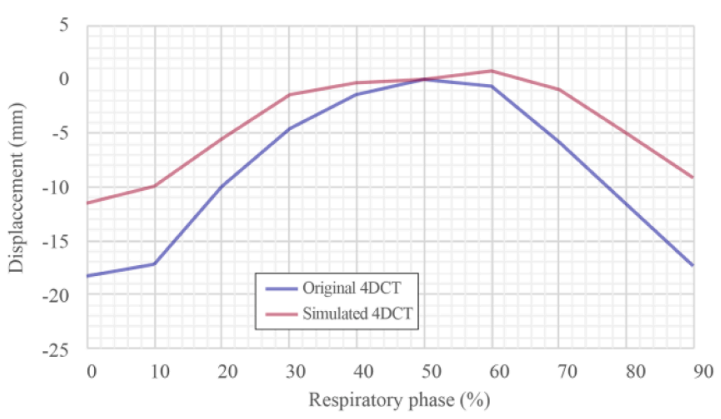

(b)

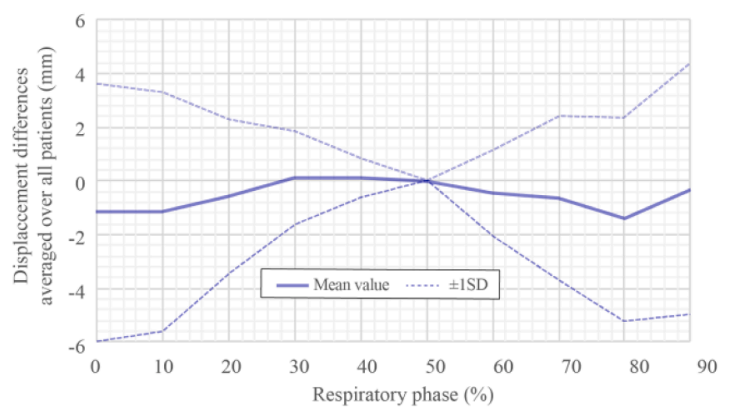

(d)

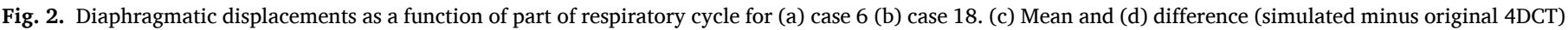
displacement averaged over all patients. Solid and dotted lines are mean \pm 1 standard deviation, respectively.

projected onto the 4D-DRR images. Training data were divided into subimages around the tumor region (some including tumor and some not). Subimage position and rotation angle were varied (data augmentation). Subimage feature information was expressed by the intensity gradient, and the tracking algorithm parameters were optimized by the extremely randomized tree method (ERT) to calculate tumor likelihood maps [9]. Use of tumor position as a function of section of the respiratory cycle allowed the creation of a regression model to generate a tumor probability estimation map.

For the prediction stage, each incoming fluoroscopic image was cropped to a subimage, and the tracking algorithm generated a tumor likelihood map and a tumor probability estimation map. This process was repeated by changing the subimage position on the incoming fluoroscopic images. The final tumor position on the fluoroscopic images was then estimated by multiplying the likelihood map by the probability map. Finally, tumor position in 3D space was derived from 2D tumor positions on a pair of fluoroscopic images.

The ground-truth tumor positions on fluoroscopic images were calculated by the markerless tracking algorithm for the training using the original 4DCT. These ground-truth positions were compared with those acquired by the tracking algorithm from the training with the simulated 4DCT. A total of 20 lung patients were randomly selected from the 40 patients evaluated for diaphragm displacement. Tracking accuracy (= "simulated 4DCT" minus "original 4DCT") was evaluated for approximately $100 \mathrm{~s}$ in mean \pm standard deviation, maximum of the absolute values. Maximum values could be affected by a single measurement point, therefore, we also evaluated the tracking accuracy in 95th percentile of the absolute value to satisfy statistical reliability. Tracking positional accuracy was expressed in the $\mathrm{X}$ (left-right), $\mathrm{Y}$ (anterior-posterior), and Z (superior-inferior) directions, and Euclidean distance was expressed according to the original 4DCT and the simulated 4DCT.

\section{Results}

\subsection{Diaphragmatic displacement}

In case 6 the diaphragmatic displacement for the simulated 4DCT was larger than that for the original for all parts of the respiratory cycle (Fig. 2a). The maximum displacement was $-21.5 \mathrm{~mm}$ and $-11.9 \mathrm{~mm}$ at T00 for the simulated and the original 4DCT, respectively. For case 18, the diaphragmatic displacement for the simulated 4DCT was larger than that for the original $4 \mathrm{DCT}$, and it was increased to $8.2 \mathrm{~mm}$ at $\mathrm{T} 90$ (Fig. 2b).

Diaphragmatic displacements averaged over all patients for the original 4DCT were slightly higher $(<1.3 \mathrm{~mm})$ than those for the simulated 4DCT; however, they were almost identical for serial sections of the respiratory cycle (Fig. 2c). Diaphragmatic displacement differences between both CTs were increased more in inhalation than exhalation, and the standard deviations were $<5.0 \mathrm{~mm}$ (Fig. 2d).

\subsection{Markerless tracking accuracy}

Simulated 4DCT for the respective sections of the respiratory cycle were successfully generated from 4DCT at T50 and appeared close to the original 4DCT (case 9) (Fig. 3a and b). Bone structures on the simulated 4DCT were not artificially deformable. The original 4DCT at T20 and T70 showed motion blur around the diaphragm; however, the diaphragm shape was clearly visible without motion blur in the simulated 4DCT because the motion blur was not included in the input CT data (4DCT at T50). To clarify, image differences from subtraction of the original 4DCT from the simulated 4DCT were minimally offset bright and dark regions.

GTV positions calculated by the simulated 4DCT were correctly overlain on the fluoroscopic images on the respective frames. GTVs are observed as dark regions outlined in yellow and orange on DFPD1 (Fig. 4a-c), whose offset shows the excursion from the T50 position (yellow).

On DFPD2, the GTV was located close to the diaphragm (Fig. 4d-f). GTV position at frame 452 was close to the exhale position (Fig. $4 \mathrm{a}$ and 
(a) Original 4DCT

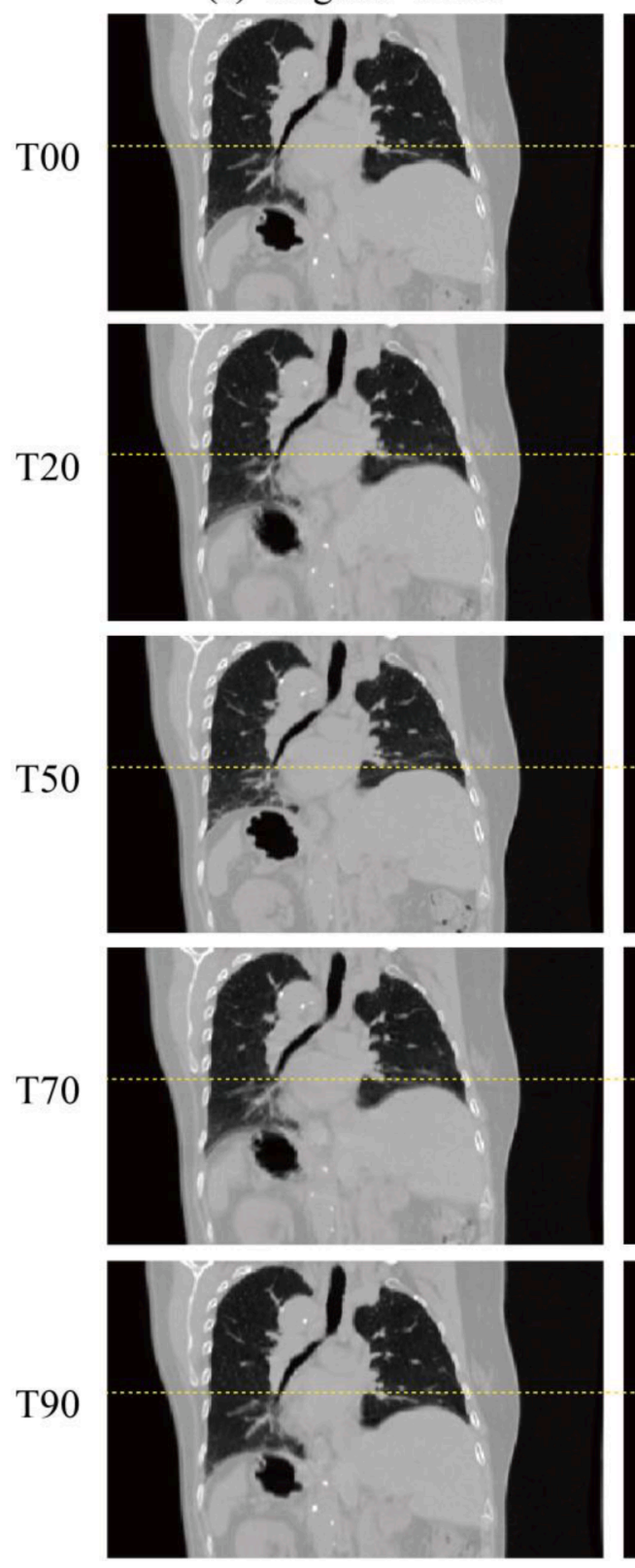

(b) Simulated 4DCT
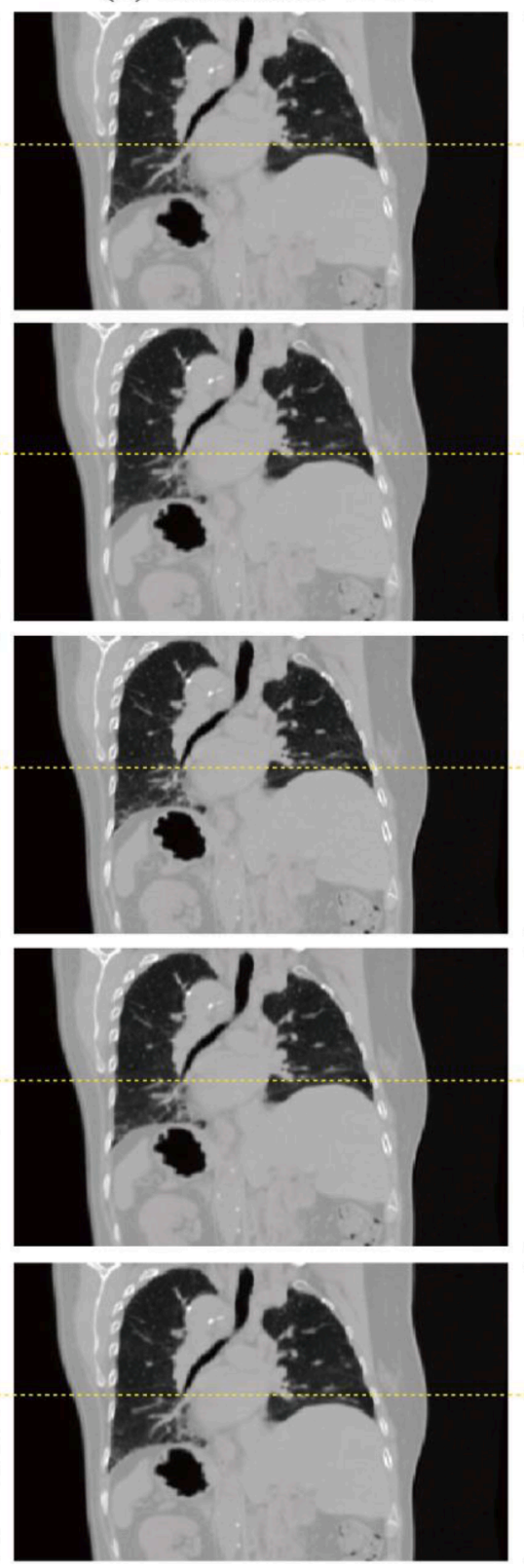

(c) Differences
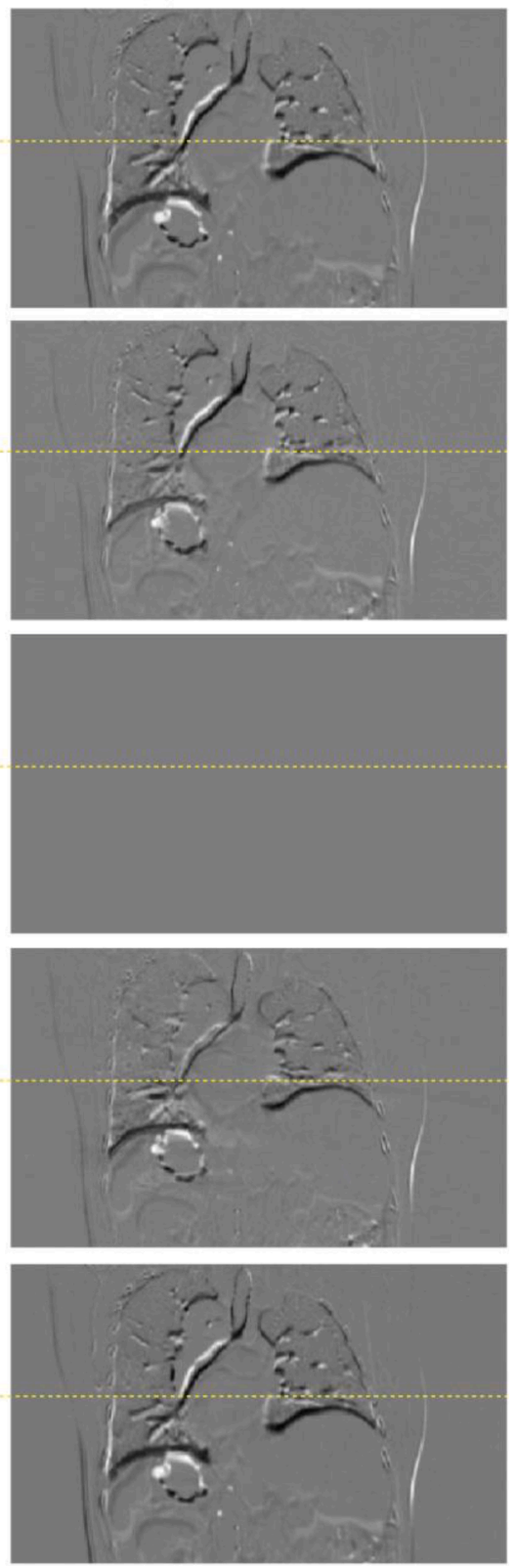

Fig. 3. (a) Original and (b) simulated 4DCT images in the coronal plane (case 9). (c) Image differences ([a] minus [b]). Yellow dotted line shows diaphragm position on the original 4DCT at T50. The patient is prone. (For interpretation of the references to colour in this figure legend, the reader is referred to the web version of this article.)

d), and the GTV moved inferiorly in frames $46 \mathrm{e}$ and 470 (Fig. 4b, c, e, and f).

The markerless tumor tracking displacement with the original 4DCT was $<5 \mathrm{~mm},<13 \mathrm{~mm}$, and $<2 \mathrm{~mm}$ in the $\mathrm{X}, \mathrm{Y}$, and $\mathrm{Z}$ directions, respectively (Fig. 5a-c). The tracking positional differences (= "simulated 4DCT" minus "original 4DCT") were $0.13 \pm 0.09 \mathrm{~mm}(\leq 0.42 \mathrm{~mm})$, $0.14 \pm 0.08 \mathrm{~mm}(\leq 0.39 \mathrm{~mm})$, and $0.25 \pm 0.15 \mathrm{~mm}(\leq 0.81 \mathrm{~mm})$ in $X, Y$, and $\mathrm{Z}$, respectively; these were much closer to those on the original 4DCT. The 95th percentile of the absolute value of displacements was $0.30 \mathrm{~mm}, 0.28 \mathrm{~mm}$, and $0.52 \mathrm{~mm}$ in X, Y, and Z, respectively (Fig. 5d).

Tracking positional errors for respective patients are summarized in Table 1. Those averaged over all patients were $0.25 \pm 0.14 \mathrm{~mm}(\leq 1.84$ $\mathrm{mm}), 0.30 \pm 0.27 \mathrm{~mm}(\leq 2.90 \mathrm{~mm})$, and $0.48 \pm 0.25 \mathrm{~mm}(\leq 2.63 \mathrm{~mm})$ for X, Y, and Z directions, respectively. The 95th percentile of the absolute value of displacements averaged over all patients was $0.56 \mathrm{~mm}$,
$0.65 \mathrm{~mm}$, and $0.96 \mathrm{~mm}$ in the $\mathrm{X}, \mathrm{Y}$ and $\mathrm{Z}$ directions, respectively.

\section{Discussion}

We generated simulated 4DCTs using DNN with multi-task learning. The quality of the simulated 4DCT was excellent. Use of the simulated 4DCT data allowed markerless tumor tracking with high accuracy (95th percentile of the absolute value: $<1 \mathrm{~mm}$ ) in the lung without actual 4DCT data, which are difficult to acquire and are accompanied by a considerable radiation dose. Diaphragm position on the simulated 4DCT was not identical to that on the original 4DCT; however, the diaphragm positions averaged over all patients were almost the same in both because the DNN is trained on many patient datasets. Namely, once a single DNN model file was trained to create simulated 4DCT, original 4DCT would not be required for every patient because simulated 4DCT is 


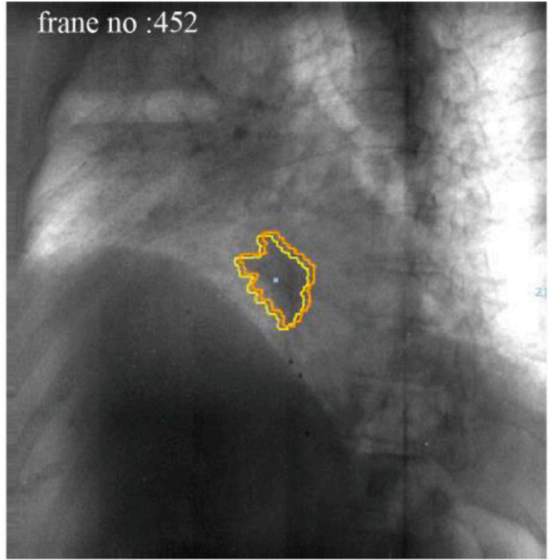

(a)

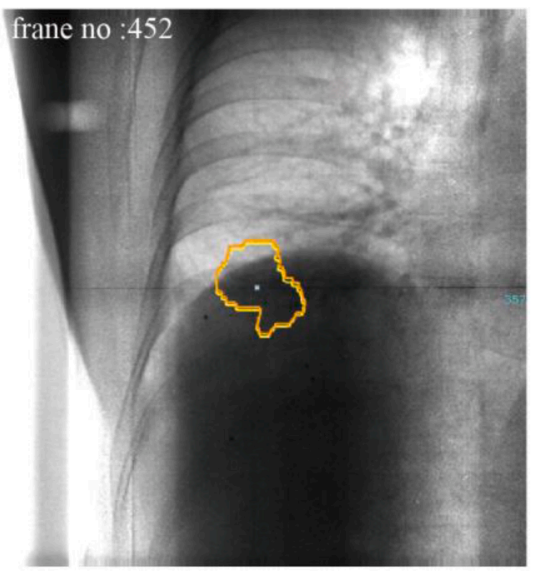

(d)

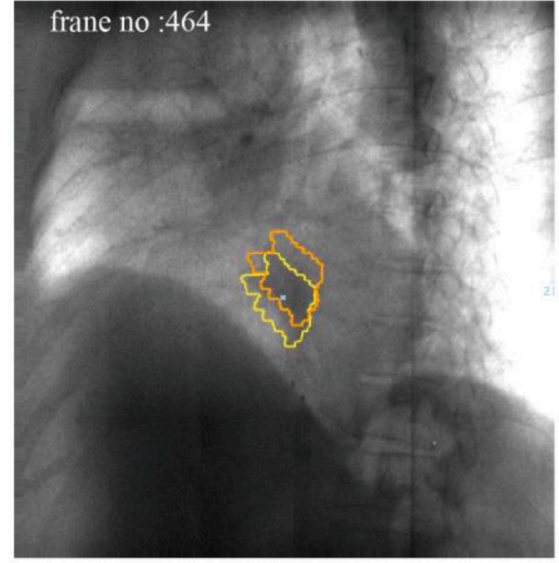

(b)

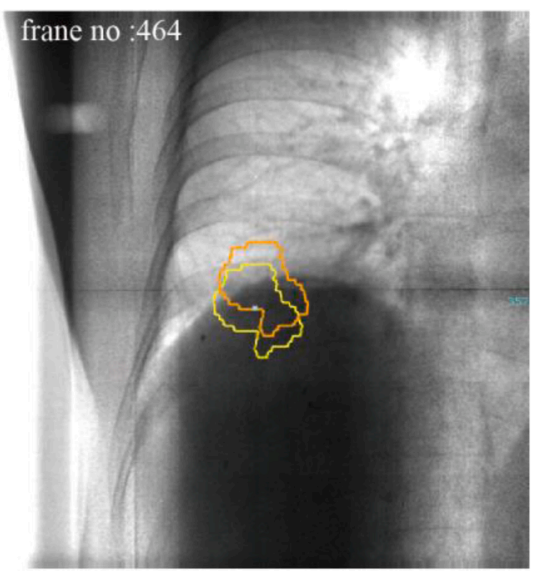

(e)

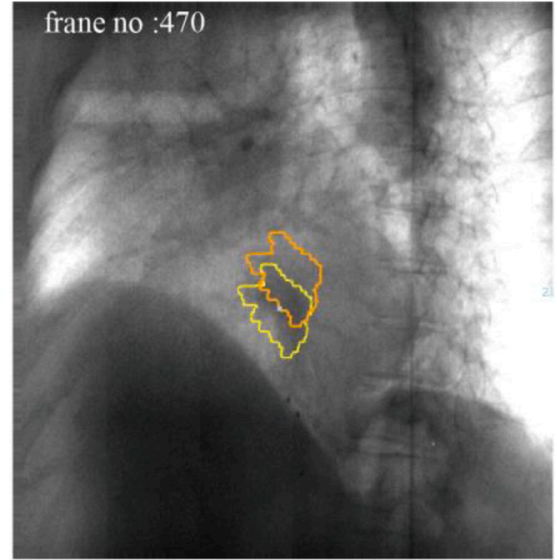

(c)

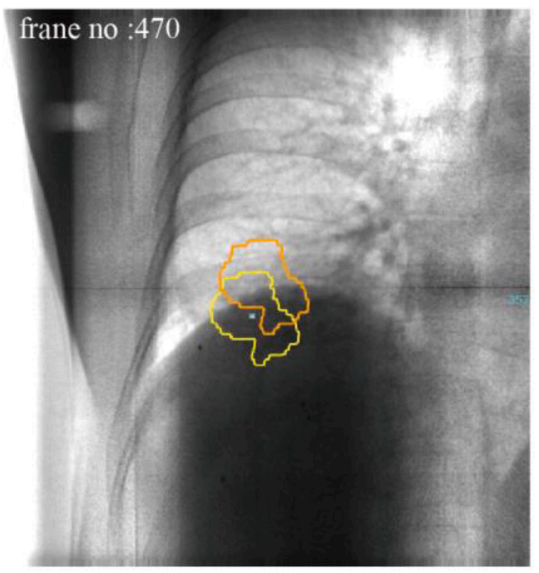

(f)

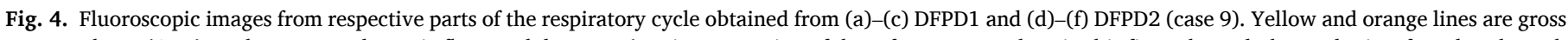

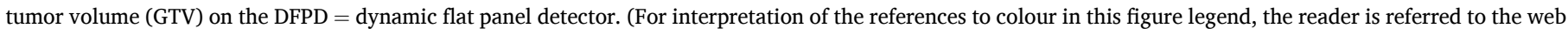
version of this article.)

used to train the markerless tracking algorithm.

Several publications have reported about image style change using DNN [26-28]; these techniques predicted similar geometrical images but different image styles. However, our DNN predicted the same image style but different geometry (from 3DCT to 4DCT); it is better for predicting deformation vector field than 4DCT. This is because gradients of deformation fields are smooth and do not require high spatial resolution compared to CT image data. The deformation vector field predicting error might be less affected than the CT image prediction. A similar concept is used in DNN for DIR [29,30].

Another major problem in radiotherapy is to preserve treatment accuracy in the presence of interfractional changes such as tumor/organ positional variations, because the planning CT cannot be used to predict them. In this study, DNN takes the 3DCT as input to generate simulate 4DCT. When we have original 4DCT data, an additional 4DCT dataset can be generated by input of 4DCT data into the DNN. For example, original 4DCT data at T00 input into the DNN will result in CT images at the maximal inhalation point. By doing this, markerless tracking model parameters would have more robustness for interfractional changes by training with original and additional 4DCT datasets.

Recent treatment planning systems have integrated 4D functions to evaluate the dose variation due to respiratory motion and perform 4D treatment planning. The former function, that of simulated organ motion in a limited region, is not yet possible. The latter function requires 4DCT data. If only 3DCT data are available, the above 4D function cannot performed. However, the ability to use simulated 4DCT data allows 4D treatment planning. While original 4DCT data are available, it is questionable whether 4DCT images can provide accurate dose distribution in actual situations due to artifact [31]. Use of the simulated 4DCT would allow artifact reduction, however, it should evaluate 4D dose distributions using original and simulated 4DCT data in the next study.

Some limitations in this study should be noted. First, it is hard to work with simulated 4DCT, because the respiratory pattern is different in every respiratory cycle. This was, however, not a problem in this study. The main purpose of this study was not focused on generating predicting 4DCT close to the original 4DCT but generating natural 4DCT.

Second, our DNN predicted deformation vector field data for respective sections of the respiratory cycle; however, cardiac motion and peristalsis, which are not related to respiration, were not predicted. These factors could affect particle beam dose distributions [32,33]. In this study, we did not compare the markerless tumor tracking accuracy using both real and simulated 4DCT data for the above motion sources.

Finally, we used simulated lung 4DCT, and we would like to apply our DNN to the abdominal region in our next study.

\section{Conclusion}

Our DNN for generating simulated 4DCT data is useful for markerless tumor tracking when original 4DCT data are not available. Use of our DNN would accelerate markerless tumor tracking and increase 


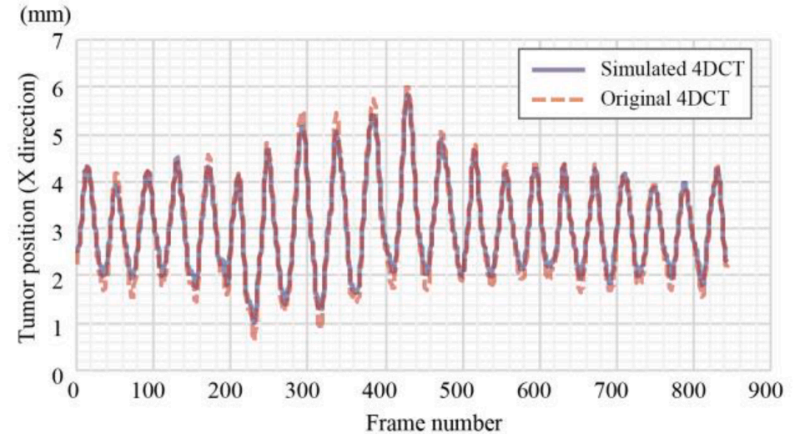

(a)

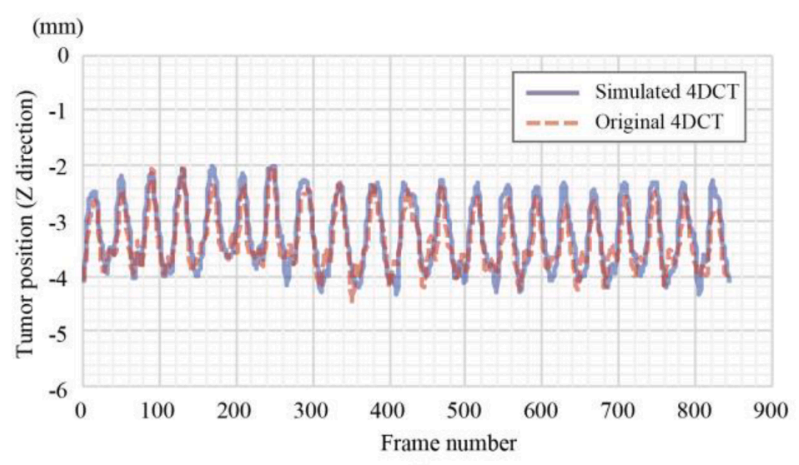

(c)

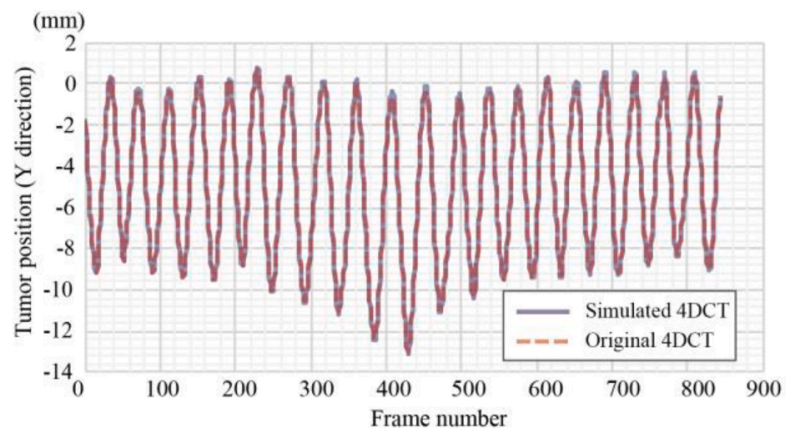

(b)

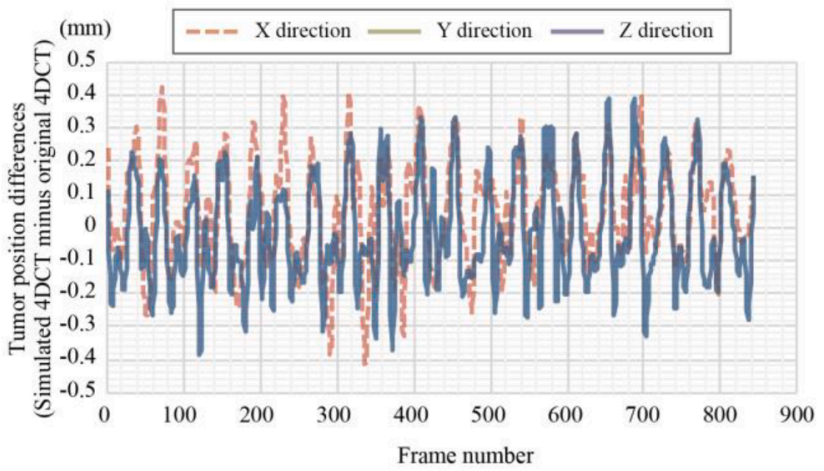

(d)

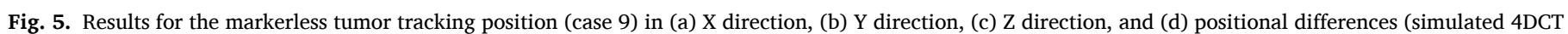
minus original 4DCT).

Table 1

Tracking positional errors (simulated 4DCT minus original 4DCT). All evaluations of metrics were of their absolute values.

\begin{tabular}{|c|c|c|c|c|c|c|c|c|c|c|c|c|c|c|c|c|c|c|c|c|}
\hline \multirow[t]{2}{*}{ No } & \multicolumn{4}{|c|}{$\mathrm{X}$ direction $(\mathrm{mm})$} & \multicolumn{4}{|c|}{ Y direction (mm) } & \multicolumn{4}{|c|}{$\mathrm{Z}$ direction (mm) } & \multicolumn{4}{|c|}{$\mathrm{R}(\mathrm{mm})$} & \multicolumn{4}{|c|}{$95 \%$ percentile $(\mathrm{mm})$} \\
\hline & Mean & \pm & SD & Max & Mean & \pm & SD & Max & Mean & \pm & SD & Max & Mean & \pm & SD & Max & $\mathrm{X}$ & $\mathrm{Y}$ & $\mathrm{Z}$ & $\mathrm{R}$ \\
\hline 1 & 0.44 & \pm & 0.35 & 1.66 & 0.55 & \pm & 0.30 & 1.83 & 0.89 & \pm & 0.63 & 2.63 & 1.27 & \pm & 0.52 & 2.85 & 1.13 & 1.07 & 2.13 & 2.40 \\
\hline 2 & 0.10 & \pm & 0.08 & 0.51 & 0.19 & \pm & 0.09 & 0.45 & 0.24 & \pm & 0.18 & 0.78 & 0.37 & \pm & 0.13 & 0.90 & 0.25 & 0.34 & 0.61 & 0.63 \\
\hline 3 & 0.21 & \pm & 0.22 & 0.92 & 0.05 & \pm & 0.06 & 0.30 & 0.14 & \pm & 0.14 & 0.62 & 0.29 & \pm & 0.24 & 0.96 & 0.69 & 0.16 & 0.41 & 0.71 \\
\hline 4 & 0.43 & \pm & 0.26 & 1.31 & 0.28 & \pm & 0.24 & 1.23 & 0.63 & \pm & 0.41 & 1.65 & 0.84 & \pm & 0.50 & 2.27 & 0.99 & 0.83 & 1.39 & 1.82 \\
\hline 5 & 0.18 & \pm & 0.14 & 0.71 & 0.28 & \pm & 0.30 & 1.12 & 0.56 & \pm & 0.40 & 1.49 & 0.71 & \pm & 0.44 & 1.65 & 0.43 & 0.92 & 1.34 & 1.54 \\
\hline 6 & 0.14 & \pm & 0.14 & 0.64 & 0.69 & \pm & 0.51 & 1.75 & 0.27 & \pm & 0.19 & 0.73 & 0.84 & \pm & 0.43 & 1.77 & 0.45 & 1.58 & 0.61 & 1.59 \\
\hline 7 & 0.38 & \pm & 0.26 & 1.14 & 1.19 & \pm & 0.77 & 2.90 & 0.66 & \pm & 0.43 & 1.90 & 1.56 & \pm & 0.64 & 3.22 & 0.84 & 2.39 & 1.43 & 2.52 \\
\hline 8 & 0.23 & \pm & 0.16 & 0.77 & 0.15 & \pm & 0.11 & 0.54 & 0.83 & \pm & 0.39 & 1.79 & 0.89 & \pm & 0.40 & 1.87 & 0.50 & 0.37 & 1.51 & 1.58 \\
\hline 9 & 0.13 & \pm & 0.09 & 0.42 & 0.14 & \pm & 0.08 & 0.39 & 0.25 & \pm & 0.15 & 0.81 & 0.34 & \pm & 0.14 & 0.85 & 0.30 & 0.28 & 0.52 & 0.59 \\
\hline 10 & 0.39 & \pm & 0.22 & 0.92 & 0.48 & \pm & 0.28 & 1.44 & 0.66 & \pm & 0.37 & 1.77 & 0.98 & \pm & 0.36 & 2.30 & 0.73 & 0.94 & 1.26 & 1.59 \\
\hline 11 & 0.18 & \pm & 0.11 & 0.58 & 0.11 & \pm & 0.08 & 0.38 & 0.62 & \pm & 0.34 & 1.55 & 0.68 & \pm & 0.31 & 1.65 & 0.38 & 0.26 & 1.12 & 1.20 \\
\hline 12 & 0.16 & \pm & 0.09 & 0.38 & 0.18 & \pm & 0.07 & 0.36 & 0.40 & \pm & 0.12 & 0.78 & 0.49 & \pm & 0.10 & 0.82 & 0.31 & 0.30 & 0.63 & 0.67 \\
\hline 13 & 0.61 & \pm & 0.52 & 1.84 & 0.37 & \pm & 0.29 & 1.50 & 0.63 & \pm & 0.28 & 1.30 & 1.07 & \pm & 0.45 & 2.38 & 1.60 & 0.97 & 1.08 & 1.95 \\
\hline 14 & 0.27 & \pm & 0.14 & 0.79 & 0.17 & \pm & 0.07 & 0.39 & 0.44 & \pm & 0.14 & 0.81 & 0.57 & \pm & 0.11 & 1.01 & 0.48 & 0.29 & 0.67 & 0.75 \\
\hline 15 & 0.12 & \pm & 0.10 & 0.47 & 0.07 & \pm & 0.05 & 0.26 & 0.42 & \pm & 0.25 & 0.98 & 0.48 & \pm & 0.22 & 0.99 & 0.32 & 0.16 & 0.84 & 0.85 \\
\hline 16 & 0.16 & \pm & 0.08 & 0.44 & 0.21 & \pm & 0.07 & 0.46 & 0.20 & \pm & 0.11 & 0.98 & 0.35 & \pm & 0.08 & 0.99 & 0.28 & 0.33 & 0.39 & 0.51 \\
\hline 17 & 0.26 & \pm & 0.17 & 0.84 & 0.32 & \pm & 0.33 & 1.17 & 0.99 & \pm & 0.37 & 1.79 & 1.14 & \pm & 0.37 & 1.89 & 0.57 & 0.97 & 1.57 & 1.66 \\
\hline 18 & 0.13 & \pm & 0.08 & 0.38 & 0.09 & \pm & 0.06 & 0.28 & 0.17 & \pm & 0.10 & 0.48 & 0.26 & \pm & 0.08 & 0.56 & 0.27 & 0.21 & 0.35 & 0.40 \\
\hline 19 & 0.13 & \pm & 0.08 & 0.36 & 0.43 & \pm & 0.07 & 0.65 & 0.29 & \pm & 0.24 & 1.02 & 0.57 & \pm & 0.16 & 1.22 & 0.29 & 0.55 & 0.70 & 0.89 \\
\hline 20 & 0.26 & \pm & 0.09 & 0.59 & 0.04 & \pm & 0.03 & 0.17 & 0.40 & \pm & 0.12 & 0.69 & 0.49 & \pm & 0.11 & 0.76 & 0.40 & 0.11 & 0.58 & 0.65 \\
\hline Total & 0.25 & \pm & 0.14 & 1.84 & 0.30 & \pm & 0.27 & 2.90 & 0.48 & \pm & 0.25 & 2.63 & 0.71 & \pm & 0.36 & 3.22 & 0.56 & 0.65 & 0.96 & 1.23 \\
\hline
\end{tabular}

Abbreviations: $\mathrm{SD}=$ standard deviation, $\mathrm{Max}=$ maximum, $\mathrm{R}=$ Euclidean distance.

treatment accuracy in thoracoabdominal treatment.

\section{Declaration of Competing Interest}

The authors declare that they have no known competing financial interests or personal relationships that could have appeared to influence the work reported in this paper.

\section{Acknowledgment}

We thank Libby Cone, MD, MA, from DMC Corp. (www.dmed.co.jp) for editing drafts of this manuscript.

\section{References}

[1] Mori S, Zenklusen S, Knopf A-C. Current status and future prospects of multidimensional image-guided particle therapy. Radiol Phys Technol 2013;6(2): 249-72. https://doi.org/10.1007/s12194-013-0199-0. 
[2] Shiinoki T, Kawamura S, Uehara T, Yuasa Y, Fujimoto K, Koike M, Sera T, Emoto Y, Hanazawa H, Shibuya K. Evaluation of a combined respiratory-gating system comprising the TrueBeam linear accelerator and a new real-time tumor-tracking radiotherapy system: a preliminary study. J Appl Clin Med Phys 2016;17(4): 202-13. https://doi.org/10.1120/jacmp.v17i4.6114.

[3] Raaymakers BW, Jurgenliemk-Schulz IM, Bol GH, Glitzner M, Kotte A, van Asselen $\mathrm{B}$, et al. First patients treated with a 1.5 T MRI-Linac: clinical proof of concept of a high-precision, high-field MRI guided radiotherapy treatment. Phys Med Biol. 2017;62:L41-L50

[4] Thongphiew D, Wu QJ, Lee WR, Chankong V, Yoo S, McMahon R, Yin F-F. Comparison of online IGRT techniques for prostate IMRT treatment: adaptive vs repositioning correction: comparison of online IGRT techniques for prostate IMRT. Med Phys 2009;36(5):1651-62. https://doi.org/10.1118/1.3095767.

[5] Lagrange JL, de Crevoisier R. [Image guided radiation therapy (IGRT)]. Bull Cancer 2010;97:857-65.

[6] Mori S, Karube M, Shirai T, Tajiri M, Takekoshi T, Miki K, Shiraishi Y, Tanimoto K, Shibayama K, Yasuda S, Yamamoto N, Yamada S, Tsuji H, Noda K, Kamada T. Carbon-ion pencil beam scanning treatment with gated markerless tumor tracking. an analysis of positional accuracy. Int J Rad Oncol Biol Phys 2016;95(1):258-66. https://doi.org/10.1016/j.ijrobp.2016.01.014.

[7] Sakata Y, Hirai R, Kobuna K, Tanizawa A, Mori S. A machine learning-based realtime tumor tracking system for fluoroscopic gating of lung radiotherapy. Phys Med Biol. 2020;65:085014.

[8] Mori S, Sakata Y, Hirai R, Furuichi W, Shimabukuro K, Kohno R, Koom WS, Kasai S, Okaya K, Iseki Y. Commissioning of a fluoroscopic-based real-time markerless tumor tracking system in a superconducting rotating gantry for carbon-ion pencil beam scanning treatment. Med Phys 2019;46(4):1561-74. https://doi.org/ 10.1002/mp.13403.

[9] Geurts P, Ernst D, Wehenkel L. Extremely randomized trees. Mach Learn 2006;63 (1):3-42. https://doi.org/10.1007/s10994-006-6226-1.

[10] Hirai R, Sakata Y, Tanizawa A, Mori S. Real-time tumor tracking using fluoroscopic imaging with deep neural network analysis. Physica Med 2019;59:22-9. https:// doi.org/10.1016/j.ejmp.2019.02.006.

[11] He K, Zhang X, Ren S, Sun J. Delving deep into rectifiers: surpassing human-level performance on ImageNet classification. In: IEEE International Conference on Computer Vision (ICCV). Santiago, Chile: IEEE; 2015. p. 1026-34.

[12] Yamamoto T, Langner U, Loo Jr BW, Shen J, Keall PJ. Retrospective analysis of artifacts in four-dimensional CT images of 50 abdominal and thoracic radiotherapy patients. Int J Rad Oncol Biol Phys 2008;72(4):1250-8. https://doi.org/10.1016/j. ijrobp.2008.06.1937.

[13] Meschini G, Vai A, Paganelli C, Molinelli S, Fontana G, Pella A, Preda L, Vitolo V, Valvo F, Ciocca M, Riboldi M, Baroni G. Virtual 4DCT from 4DMRI for the management of respiratory motion in carbon ion therapy of abdominal tumors. Med Phys 2020;47(3):909-16. https://doi.org/10.1002/mp.13992.

[14] Miyabe Y, Narita Y, Mizowaki T, Matsuo Y, Takayama K, Takahashi K, Kaneko S, Kawada N, Maruhashi A, Hiraoka M. New algorithm to simulate organ movement and deformation for four-dimensional dose calculation based on a threedimensional CT and fluoroscopy of the thorax: algorithm to simulate organ movement and deformation. Med Phys 2009;36(10):4328-39. https://doi.org/ 10.1118/1.3213083.

[15] Mori S. Real-time image-processing algorithm for markerless tumour tracking using X-ray fluoroscopic imaging. Br J Radiol 2014;87(1037):20140001. https:// doi.org/10.1259/bjr.20140001.
[16] Bishop CM. Neural networks for pattern recognition. Oxford University Press; 1995.

[17] Junyuan X, Linli X, Enhong C. Image denoising and inpainting with deep neural networks. Adv Neural Inf Process Syst 2012:341-9.

[18] Jain V, Seung S. Natural image denoising with convolutional networks. Adv Neural Inf Process Syst 2009:769-76.

[19] Zhang K, Zuo W, Chen Y, Meng D, Zhang L. Beyond a Gaussian denoiser: residual learning of deep CNN for image denoising. IEEE Trans Image Process 2017;26(7): 3142-55. https://doi.org/10.1109/TIP.2017.2662206.

[20] Vincent P, Larochelle H, Bengio Y, Manzagol P-A. Extracting and composing robust features with denoising autoencoders. In: Proceedings of the 25th international conference on machine learning; 2008. p. 1096-103.

[21] Sergey I, Christian S. Batch Normalization: accelerating deep network training by reducing internal covariate shift. In The 32nd International Conference on Machine Learning 2015:448-56.

[22] Ronneberger O, Fischer P, Brox T. U-Net: convolutional networks for biomedical image segmentation. In: Medical Image Computing and Computer-Assisted Intervention (MICCAI). Springer; 2015. p. 234-41.

[23] Radford A, Metz L, Chintala S. Unsupervised representation learning with deep convolutional generative adversarial networks. In International Conference on Learning Representations 2016.

[24] Sharp GC, Kandasamy N, Singh H, Folkert M. GPU-based streaming architectures for fast cone-beam CT image reconstruction and demons deformable registration. Phys Med Biol 2007;52(19):5771-83. https://doi.org/10.1088/0031-9155/52/ 19/003.

[25] Sener O, Kotltun V. Multi-task learning as multi-objective optimization. arXiv: 181004650.

[26] Gatys L, Ecker A, Bethge M. A neural algorithm of artistic style. J Vis 2016;16(12): 326. https://doi.org/10.1167/16.12.326.

[27] Li Y, Liu M-Y, Li X, Yang M-H, Kautz J. A closed-form solution to photorealistic image stylization. arXiv e-prints2018.

[28] Xiao H. MR-based synthetic CT generation using a deep convolutional neural network method. Med Phys 2017;44(4):1408-19. https://doi.org/10.1002/ mp. 12155.

[29] de Vos BD, Berendsen FF, Viergever MA, Sokooti H, Staring M, Isgum I. A deep learning framework for unsupervised affine and deformable image registration. Med Image Anal 2019;52:128-43. https://doi.org/10.1016/j.media.2018.11.010.

[30] Kim B, Kim J, Lee J-G, Kim DH, Park SH, Ye JC. Unsupervised deformable image registration using cycle-consistent CNN. arXiv e-prints 2019.

[31] Mori S, Hirai R, Sakata Y. Using a deep neural network for four-dimensional CT artifact reduction in image-guided radiotherapy. Physica Med 2019;65:67-75. https://doi.org/10.1016/j.ejmp.2019.08.008.

[32] Kumagai M, Hara R, Mori S, Yanagi T, Asakura H, Kishimoto R, et al. Impact of intrafractional bowel gas movement on carbon ion beam dose distribution in pancreatic radiotherapy. Int J Rad Oncol Biol Phys 2009;73(4):1276-81. https:// doi.org/10.1016/j.ijrobp.2008.10.055.

[33] Mori S, Chen GT, Endo M. Effects of intrafractional motion on water equivalent pathlength in respiratory-gated heavy charged particle beam radiotherapy. Int $\mathrm{J}$ Rad Oncol Biol Phys 2007;69(1):308-17. https://doi.org/10.1016/j. ijrobp.2007.05.018. 